\title{
Anderson and Krathwohl's Two-Dimensional Taxonomy Applied to Supporting and Predicting Learning During Search
}

\author{
Kelsey Urgo \\ School of Information and Library Science \\ University of North Carolina at Chapel Hill \\ [kurgo@unc.edu]
}

\begin{abstract}
There is a growing body of research in the Search as Learning community that recognizes the need for users to learn during search, but modern search systems have yet to adapt to support this need. Our research proposes three research goals toward addressing the support of user learning during search. Research goal 1 (RG1) introduces a more precise and reliable metric of assessing user learning. Anderson \& Krathwohl's 2-dimensional taxonomy is used as a framework to develop learning objectives and assessment questions to measure user learning during search. Additionally, Anderson \& Krathwohl's taxonomy is used as a coding scheme to outline the pathways users traverse along the way to a particular learning objective. Research goal 2 (RG2) investigates the prediction of learning objectives using behavioral measures. Finally, research goal 3 (RG3) proposes a search system that presents information relevant to the user based on their current learning sub-goal and scaffolds information based on the pathways they are likely to traverse given a particular learning objective.
\end{abstract}

\section{KEYWORDS}

search as learning; learning assessment; predicting learning; search behavior

\section{ACM Reference Format:}

Kelsey Urgo. 2020. Anderson and Krathwohl's Two-Dimensional Taxonomy Applied to Supporting and Predicting Learning During Search. In 2020 Conference on Human Information Interaction and Retrieval (CHIIR '20), March 14-18, 2020, Vancouver, BC, Canada. ACM, New York, NY, USA, 4 pages. https://doi.org/10.1145/3343413.3377947

\section{INTRODUCTION}

Learning is a common process during search and an important outcome of search. Modern search systems, however, have been optimized for simple lookup tasks and often lack the support, feedback, and design necessary for effective user learning. In response to this need, work in the Search as Learning community has focused efforts around better understanding human learning, measuring learning and learning outcomes during search, and designing systems that promote learning [7].

The proposed research investigates learning during search. In particular, we focus on the three following research goals.

Permission to make digital or hard copies of part or all of this work for personal or classroom use is granted without fee provided that copies are not made or distributed for profit or commercial advantage and that copies bear this notice and the full citation on the first page. Copyrights for third-party components of this work must be honored.

For all other uses, contact the owner/author(s).

CHIIR '20, March 14-18, 2020, Vancouver, BC, Canada

(c) 2020 Copyright held by the owner/author(s).

ACM ISBN 978-1-4503-6892-6/20/03.

https://doi.org/10.1145/3343413.3377947
RG1: The first research goal investigates effective defining and assessment of user learning objectives during search. In order to design a system that effectively supports learning during search, we must first better understand and assess learning of users. In the field of education, Anderson \& Krathwohl's two-dimensional taxonomy of learning was developed to help educators more precisely measure learning objectives [1]. In the context of interactive information retrieval (IIR), researchers have used this taxonomy to investigate how cognitive processes associated with search tasks influence users' needs, behaviors, and experiences [2, 6, 11-13, 16]. This application of A\&K's 2D taxonomy has been exclusively along a single dimension, the cognitive process dimension. We extend the application of A\&K's 2D taxonomy to include both dimensions of the taxonomy, the cognitive process dimension and the knowledge dimension. Using both dimensions allows us to more precisely define and articulate both the user learning objectives and the assessment of user learning. A\&K's 2D taxonomy also checks the alignment of the learning objective and assessment to ensure assessment questions correspond to what a user is intending to learn.

Our first research goal will use A\&K's 2D taxonomy to design search learning tasks and assessment questions that correspond to a particular cognitive process and knowledge type combination. Some prior work has used A\&K's 2D taxonomy to score written summaries and test questions $[8,15]$. Our research will extend this work by using both dimensions of A\&K's 2D taxonomy to categorize assessment questions into a particular cell. We also use A\&K's taxonomy to ensure the alignment of assessment and learning objective (search learning task). Using both dimensions of A\&K's 2D taxonomy to design search learning tasks and assessment questions will allow us to control for the knowledge dimension. This will help us to better understand the strength of the effects found (e.g., perceived difficulty) in prior work on cognitive processes. Perhaps effects grows stronger as we move down the knowledge dimension? We will also investigate the knowledge dimension itselfunderstanding the effects of the knowledge dimension on outcomes related to: (1) search behaviors, (2) types of information, (3) perceived difficulty, and (4) learning.

Additionally, A\&K's 2D taxonomy will be applied as a coding scheme to categorize user's search session think-aloud comments. This will allow us to better understand the ways users break learning objectives into sub-goals. Each learning unit or learning need transition will be mapped to a particular cell in A\&K's taxonomy to reveal the learning pathways traversed by searchers based on their specific learning objective.

RG2: The second research goal (RG2) will investigate predicting a user's learning objective during search. Understanding a user's learning end goal before they reach the end of their search session 
would allow a system to provide support specific to their learning need. Learning objectives will be defined by tasks that have been mapped to a knowledge type and cognitive process cell in A\&K's 2-D taxonomy. We will generate behavioral measures to develop and evaluate models that predict a user's specific learning objective.

RG3: The third research goal (RG3) will be to develop and evaluate a search system that presents information to support user learning. Current search systems are effective at simple lookup tasks but lack supportive structure useful for user learning. In order to better support learning during search we will develop a system that provides relevant and scaffolded information based on a user's particular learning objective or current learning sub-goal. This system will use information from RG2 that correlates a particular learning objective with a particular information type. For example, if a user is engaged in a learning objective involving procedural knowledge, the system would present relevant video tutorials given that procedural knowledge learning objectives are found to be correlated with video information type. Prior work in self-regulated learning has shown the importance of setting sub-goals for successful learners [3]. Given the importance of sub-goals in learning, our system will provide scaffolding for the user. Using pathways data from RG1, the system will scaffold a learning objective into commonly traversed sub-goals or cells in A\&K's 2D taxonomy.

\section{BACKGROUND}

Our proposed research applies Anderson \& Krathwohl's 2-D taxonomy and builds on three areas of prior work. First, we build on work in IIR that has studied cognitive complexity. Second, we build on prior work that has investigated predicting knowledge gains. Finally, we build on studies of self-regulated learning, which aim to understand the qualities and mental activities of effective learners.

Anderson \& Krathwohl 2-D Taxonomy: Anderson \& Krathwohl's 2-dimensional taxonomy [1] comes from the field of education. A\&K's 2-D taxonomy was developed to better define and articulate learning objectives and the activities and assessment aligned with such objectives. A\&K's 2-D taxonomy is comprised of a cognitive process dimension and a knowledge type dimension. The cognitive process dimension contains six levels of increasing complexity-(1) remember (recalling information), (2) understand (constructing meaning), (3) apply (using information), (4) analyze (breaking information into parts), (5) evaluate (judging information based on criteria), and (6) create (novel form of information). The knowledge type dimension contains four levels ranging from the most concrete to the most abstract-(1) factual (basic information elements), (2) conceptual (larger framework and relationships of information), (3) procedural (steps of how to do something), and (4) metacognitive (knowledge of cognition and self-knowledge).

Cognitive Complexity: Cognitive complexity, in terms of IIR, refers to the various mental activities that are required to complete a given learning-intensive search task. The work of Jansen et al. [12] first introduced the application of A\&K's taxonomy to develop search tasks along varying cognitive complexity levels. Results found that participants expended the most effort during tasks associated with the mid-complex levels (i.e., apply and analyze). For these tasks, participants visited more pages, issued more queries, issued longer queries, and had longer completion times. One possibility is that participants satisficed during the most complex tasks of evaluate and create.

A\&K's taxonomy was also applied in the work of Wu et al. [16] to develop 20 search tasks which varied along 5 cognitive process levels (the apply cognitive level was not included). A subset of 10 of the developed tasks were used in a lab study to investigate the effects of complexity on user behaviors. Mid-complexity tasks were found to be more engaging, potentially because tasks of mid-level cognitive complexity tend to be mid-range in difficulty. During tasks of higher complexity, such as evaluate and create, participants exhibited more search activity and participants reported greater levels of perceived difficulty. Kelly et al. [13] used all 20 search tasks from $\mathrm{Wu}$ et al. [16] in a subsequent study and confirmed those trends from $\mathrm{Wu}$ et al. [16]. Additionally, during complex tasks participants had more divergent search strategies compared to each other. Also using a subset of tasks developed in Wu et al. [16], Capra et al. [6] introduced a search assistance tool that displayed search trails of participants who had previously completed that same task. This study investigated the effects of task complexity on participants' use of this search assistance tool. Results found that complex tasks had greater use of the tool. Brennan et al. [5] studied how task complexity affects searchers with different cognitive abilities. One cognitive ability, associated memory (AM), had an interaction effect with task complexity. Participants in the high-AM group experienced similar levels of time pressure irrespective of task complexity. Conversely, low-AM participants experienced greater time pressure during complex versus simple tasks.

Learning Assessment: Prior work in IIR has used various methods of knowledge gain measurement to address open questions in the Search as Learning community regarding user learning during search. Wilson and Wilson [15] assessed participants' knowledge gains by scoring participants' post-task written summaries. A subset of the codes (D-Qual, D-Intrp, and D-Crit) were developed using cognitive process levels from A\&K's taxonomy. D-Qual (understand cognitive process) and D-Intrp (analyze cognitive process) codes detected knowledge gains more effectively. The imposed time limit (5 minutes) in which to write summaries may have affected the D-Crit (evaluate cognitive process) code's precision in detecting knowledge gains. Collins-Thompson et al. [8] created six open-ended questions (Q1-Q6) for a particular task, each question corresponding to a single cognitive process in A\&K's 2D taxonomy. Participant answers to questions were scored for exhibiting factual versus conceptual knowledge types. Tasks categorized as easy showed participant knowledge gains of "low-level learning" (Q1-Q3) and tasks categorized as more difficult showed more "high-level learning" (Q4-Q6). Gadiraju et al. [9] measure knowledge gains of participants using pre- and post-task assessments with answer choices: true, false, and I don't know. Time spent reading pages and complex query-terms were positively correlated with knowledge gains.

Self-Regulated Learning: Prior work in self-regulated learning has recognized several characteristics of effective self-regulated learners. Azevedo et al. [3] created a qualitative coding scheme to identify characteristics of so-called "high jumpers" who acquired more complex mental models of a given subject while gathering information in a hypermedia environment. High jumpers planned 
learning through the creation of sub-goals, activated prior knowledge, monitored their understanding throughout the session, and planned their time.

Biswas et al. [4] discusses scaffolding in Open-Ended Learning Environments (OELEs) to support novice learners. These scaffolds are defined as "actions taken by the learning environment, based on the learner's interactions, intended to support the learner in completing a task and understanding the topic." [4, p.1]. Moos [14] discusses various approaches to scaffolding. Recent insights in scaffolding highlight the need for individualized assistance that provides ongoing diagnosis, support, and feedback throughout a given learning task. Also, effective scaffolding requires that assistance decreases as a student's competence increases.

\section{PHASE 1: UNDERSTANDING AND PREDICTING LEARNING}

Phase 1 of research will consist of two distinct studies. Study 1 will address RG1 using A\&K's 2D taxonomy to develop and operationalize search learning tasks (learning objectives), assessment of learning acquired during search, and mapping of user think-aloud comments to sub-goals along a learning pathway. Study 2 addresses RG2 and predict user learning objectives using behavioral measures.

Study 1 - Learning Pathways: Study 1 will aim to understand(1) how a learning objective affects outcomes such as search behavior and learning and (2) how users sub-divide learning objectives into smaller sub-goals (associated with specific cells in A\&K's 2-D taxonomy) that are either planned or serendipitous. Our goal is to uncover common pathways traversed by users based on their ultimate learning objectives. Gaining such insights will enable us to develop search systems and tools that provide scaffolding and encourage learning.

Anderson \& Krathwohl frame learning objectives as a verb-noun pair [1]. Here, we consider our search learning task to be a learning objective, thus also a verb-noun pair. The verb of the search learning task maps to the cognitive level in the taxonomy and the noun maps to the knowledge type. For example, "Run a mergesort on this set of integers: $\{9,2,6,3,8,1,4,7\}$." This search learning task is categorized as apply cognitive process and procedural knowledge. The verb run indicates using knowledge or apply and the noun mergesort indicates a series of steps or procedural knowledge.

We define learning pathways as a series of sub-goals that users encounter on the way to their specific learning objective. For example, a user might have the following learning objective "judge whether mergesort or heapsort is most efficient and explain why." During a search session for this learning objective a user unfamiliar with the topic may break apart their search process into the following sub-goals: (1) gather information about steps of a heapsort (understand procedural), (2) gather information about steps of mergesort (understand procedural), (3) gather information about a newly encountered concept "divide and conquer" algorithm (understand conceptual), (4) try running a mergesort algorithm (apply procedural), and (5) find articles that distinguishes mergesort from heapsort (analyze procedural). At the final step (5) the user finds an article with a graph that shows the efficiency of mergesort being greater than that of heapsort and is able to arrive at a judgment choosing mergesort as the more efficient algorithm. This pathway contains five subgoals and covers four unique cells of A\&K's taxonomy in route to the final evaluate procedural learning objective.

The study protocol will proceed as follows. Participants will complete three tasks of varying complexity and knowledge type. During each task, participants will be asked to search for information and take notes. Next, participants will review their notes and produce a verbal response to the task's main question, which will be video recorded. Participants will complete pre- and post-task questionnaires before and after each task. The pre-task questionnaire is designed to measure interest and prior knowledge, task understanding and a priori determinability, expected difficulty in search and video assessment, and perceptions of cognitive processes and knowledge types. The post-task questionnaire is designed to measure interest, perceived knowledge increase, experienced difficulty and satisfaction in search and video assessment, and perceptions of cognitive processes and knowledge types engaged in during search. Finally, we will give an assessment that asks various questions covering five of the cognitive processes of all three tasks to assess depth of knowledge across topics.

The study will investigate four main research questions. To reiterate, participants will complete learning-intensive search tasks associated with a specific cognitive process and knowledge type in A\&K's taxonomy. RQ1 investigates the effects of the task's knowledge type and cognitive process (learning objective) on outcomes related to: (1) search behaviors, (2) types of information used (e.g., there may be a correlation between procedural knowledge and video demonstrations), (3) perceived difficulty, and (4) learning. To measure learning, participants will complete post-task tests designed to measure knowledge gains associated with specific knowledge types and cognitive processes along A\&K's taxonomy. RQ2 investigates the learning pathways. What are the regularities or frequencies of pathways (series of sub-goals) users traverse during a search session? We will analyze these pathways as a series of transition probabilities from one cell (sub-goal) to another cell in A\&K's taxonomy. RQ3 investigates the effect of the learning objective (knowledge type and cognitive process combination) on the learning pathways. Perhaps less complex cognitive processes (e.g., remember) elicit shorter (and more straightforward) pathways along the taxonomy than more complex cognitive processes (e.g., evaluate). A secondary question of RQ3-How do user characteristics affect learning pathways? Perhaps domain knowledge impacts a user's starting point in A\&K's taxonomy, e.g., a novice may start in understand conceptual where an expert may start in analyze conceptual. Finally, RQ4 investigates the effects of the pathway on learning conditioned on a particular learning objective. We hypothesize, given a fixed learning objective, users who traverse fewer cells might experience lower knowledge gains than those who traverse a greater number of cells.

To address these questions various data will be collected and analyzed. Pre-/post-questionnaire data will be analyzed along with the scored (using a key of predetermined correct answers) task assessment questions. Think-aloud comments indicative of subgoal shifts will be coded using A\&K's 2D taxonomy as a particular cognitive process/knowledge type combination. System interaction data (e.g., queries) will be collected across search sessions.

Study 2 - Predicting Learning Objectives: The second study will target RG2, investigating the prediction of learning objectives 
using behavioral measures captured during search sessions. Search tasks will be designed using A\&K's 2D taxonomy with varying cognitive processes and knowledge types.

The data collection will be conducted using Amazon's Mechanical Turk (MTurk). Participants will be given a search task and access to a live search engine. All user interactions with the search service will be logged on our server. This user interaction data will be included in the behavioral measures used to develop and evaluate models that predict learning objectives corresponding to a particular cell in A\&K's 2D taxonomy.

The experimental protocol will be as follows. After reading the task, participants will complete a pre-task questionnaire designed to measure the participant's level of prior knowledge in the task domain, interest, and perceptions of determinability (i.e., lack of uncertainty regarding the task requirements, processes involved, and form of the solution). Participants will be asked to complete search tasks oriented toward a learning objective that fits a specific cell in the taxonomy. Upon completing the search task, participants will be given a single short-answer assessment question to better understand what the user learned from the search session followed by a post-task questionnaire asking about their level of engagement throughout the search session and their retrospective assessment of the task difficulty.

This study will target one main research question. What are behavioral indicators that are predictive of a user's learning objective (knowledge type and cognitive process combination)? We will consider different types of evidence including-amount of information, trial and error, types of information, and temporal characteristics.

\section{PHASE 2: SYSTEM-SUPPORTED LEARNING}

Prior work in self-regulated learning has highlighted the importance of goal-setting and system scaffolding for successful user learning $[3,10]$. In Phase 2, RG3 will be targeted through the development of a system that (1) presents types of information found to be relevant for a particular learning objective and (2) scaffolds information into sub-goals relevant to a particular learning objective. Addressing the first target, the system will present users with types of information found (in RG2) to be predictive of the particular learning goal a user is currently investigating. For example, if a user is investigating an understand conceptual learning goal then encylopedic articles may be the most predominant search result. To address the second target, the system will present a user with sub-goals related to the pathway found from RG1 to be typical for a given learning objective. For example, if a learning objective is known to have the pathway: understand conceptual, understand procedural, apply procedural; then the system could present information types and keywords relevant to each sub-goal in sequence. The main research question for Phase 2 investigates the effect the new learning-supportive system has on user learning. Does learning increase in our system versus a traditional system?

\section{DISCUSSION}

Our research has three main objectives-(RG1) introduce a more precise and reliable metric of assessing user learning; (RG2) investigate the prediction of learning objectives using behavioral measures; and (RG3) propose a search system that supports user learning. In RG1, we will investigate assessing user learning in two ways. First, we will develop targeted learning assessment questions aligned with cells of A\&K's taxonomy. Second, we will investigate the learning pathways (sub-goals) users traverse given a particular learning objective (knowledge type and cognitive process). In RG2, we will use behavioral measures to predict learning objectives. We will also explore information types associated with different learning objectives. Finally, in RG3, we will use information from RG1 and RG2 to develop a learning supportive search system. The proposed system will present information types and keywords (from RG2) associated with a user's current learning objective. Additionally, findings from RG1 will inform how the proposed system might scaffold user learning based on the pathways (i.e., cells in A\&K's taxonomy) a user is likely to traverse given a particular learning objective.

Acknowledgements: This work was supported by NSF grant IIS-1718295. Any opinions/findings are those of the authors, not necessarily the sponsors.

\section{REFERENCES}

[1] Lorin W Anderson and David R Krathwohl. 2001. A taxonomy for learning, teaching, and assessing: A revision of Bloom's taxonomy of educational objectives, complete edition.

[2] Jaime Arguello. 2014. Predicting Search Task Difficulty. In ECIR. Springer, 88-99.

[3] Roger Azevedo, John T. Guthrie, and Diane Seibert. 2004. The Role of SelfRegulated Learning in Fostering Students' Conceptual Understanding of Complex Systems with Hypermedia. Fournal of Educational Computing Research 30, 1-2 (Jan. 2004), 87-111. https://doi.org/10.2190/DVWX-GM1T-6THQ-5WC7

[4] Gautam Biswas, Roger Azevedo, Valerie Shute, and Susan Bull. 2013. Workshop on Scaffolding in Open-Ended Learning Environments (OELEs). In International Conference on Artificial Intelligence in Education. Springer, 952-953.

[5] Kathy Brennan, Diane Kelly, and Jaime Arguello. 2014. The effect of cognitive abilities on information search for tasks of varying levels of complexity. In IIIX. ACM, 165-174.

[6] Robert Capra, Jaime Arguello, Anita Crescenzi, and Emily Vardell. 2015. Differences in the use of search assistance for tasks of varying complexity. In SIGIR. ACM, 23-32.

[7] Kevyn Collins-Thompson, Preben Hansen, and Claudia Hauff. 2017. Search as Learning (Dagstuhl Seminar 17092). (2017). https://doi.org/10.4230/dagrep.7.2.135

[8] Kevyn Collins-Thompson, Soo Young Rieh, Carl C. Haynes, and Rohail Syed. 2016. Assessing Learning Outcomes in Web Search: A Comparison of Tasks and Query Strategies. In CHIIR. ACM, 163-172.

[9] Ujwal Gadiraju, Ran Yu, Stefan Dietze, and Peter Holtz. 2018. Analyzing Knowledge Gain of Users in Informational Search Sessions on the Web. In CHIIR.

[10] Jeffrey Alan Greene, Jane Robertson, and Lara-Jeane Croker Costa. 2011. Assessing self-regulated learning using think-aloud methods. (2011), 313-328.

[11] Xiao Hu and Noriko Kando. 2017. Task complexity and difficulty in music information retrieval. JASIST 68, 7 (2017), 1711-1723.

[12] Bernard J Jansen, Danielle Booth, and Brian Smith. 2009. Using the taxonomy of cognitive learning to model online searching. IP\&M 45, 6 (2009), 643-663.

[13] Diane Kelly, Jaime Arguello, Ashlee Edwards, and Wan-Ching Wu. 2015. Development and evaluation of search tasks for IIR experiments using a cognitive complexity framework. In ICTIR. ACM, 101-110.

[14] DC Moos. 2017. Emerging classroom technology: Using self-regulation principles as a guide for effective implementation. Handbook of self-regulation of learning and performance (2017), 243-253.

[15] Mathew J Wilson and Max L Wilson. 2013. A comparison of techniques for measuring sensemaking and learning within participant-generated summaries. FASIST 64, 2 (2013), 291-306.

[16] Wan-Ching Wu, Diane Kelly, Ashlee Edwards, and Jaime Arguello. 2012. Grannies, tanning beds, tattoos and NASCAR: Evaluation of search tasks with varying levels of cognitive complexity. In II iX. ACM, 254-257. 